\title{
Una mirada a la discapacidad psicosocial desde las ciencios humanas, sociales Y de la salud
}

\author{
Andrea del Pilar Arenas* \\ Didier Evander Melo-Trujillo**
}

Recibido en 08 de julio de 2019, aceptado en 26 de agosto de 2020

Citar este artículo así: Arenas AP, Melo-Trujillo DE. Una mirada a la discapacidad psicosocial desde las ciencias humanas, sociales y de la salud. Hacia. Promoc. Salud. 2021; 26 (1): 69-83. DOI: 10.17151/hpsal.2021.26.1.7

\section{Resumen}

La enfermedad mental es un tema enmarcado por el estigma que socialmente relega y signa al sujeto, advirtiendo de un tipo de discapacidad antes equiparado con la intelectual: la discapacidad psicosocial, hoy formalmente reconocida por la Organización Mundial de la Salud. Objetivo. Comprender la concepción de enfermedad mental y discapacidad psicosocial, analizando el estigma y la exclusión como aspectos sustantivos que configuran dicho tipo de discapacidad. Materiales y Métodos. Estudio cualitativo de tipo descriptivo para el cual se aplicó en dos fases una entrevista abierta estructurada y una encuesta a 51 personas: 20 profesionales de las ciencias humanas, sociales y de la salud y 31 estudiantes de psicología residentes en la ciudad de Ibagué. Resultados. Tanto desde la percepción de estudiantes como de profesionales, la enfermedad mental y su abordaje se remiten a los postulados del modelo médico tradicional. Existe desconocimiento de la discapacidad psicosocial, así como de los aspectos diferenciadores y articuladores de ésta con la enfermedad mental. Los participantes refieren que tanto la enfermedad mental como cualquier tipo de discapacidad están rodeadas de prejuicios y estereotipos que excluyen y por tanto discriminan. Conclusión. Profesionales y estudiantes evidencian ausencia de información, formación y sensibilización sobre el tema, por lo que enfermedad mental y discapacidad psicosocial son dos términos poco relacionados en el contexto de la investigación. Urge en el abordaje de la temática la articulación modelo médico/modelo social dando relevancia al papel que juega el contexto en la relación sujeto/sociedad desde un enfoque de derechos.

\section{Palabras clave}

Salud Mental, Discapacidad, Psicosocial, Exclusión social, Enfermedad mental, Estigma (fuente: DeCS, BIREME).

\footnotetext{
* Psicóloga. Magister en Intervención Social en las Sociedades del Conocimiento. Universidad Nacional Abierta y a Distancia UNAD. Ibagué Colombia. andrea.arenas@unad.edu.co.Autor de correspondencia. (D) orcid.org/0000-0001-7920-0424. Google

** Psicólogo. Universidad Nacional Abierta y a Distancia UNAD. Ibagué. Colombia. demelot@unadvirtual.edu.co

(D) orcid.org/0000-0003-2492-186X. Google
} 


\title{
A look at the psychosocial disability from the human, social and health sciences
}

\begin{abstract}
Mental illness is an issue framed by stigma that socially relegates and marks the subject, warning of a type of disability previously equated with intellectual disability: psychosocial disability nowadays formally recognized by the World Health Organization. Objective. To understand the concept of mental illness and psychosocial disability, analyzing stigma and exclusion as substantive aspects that make up this type of disability. Materials and Methods. Descriptive, qualitative study for which an open structured interview and a survey of 51 people were applied in two phases: 20 professionals of the human, social and health sciences and 31 psychology students residing in the city of Ibagué. Results. Both, from the perspective of students and professionals, consider that mental illness and its approach are referred to the postulates of the traditional medical model. There is a lack of knowledge about psychosocial disability, as well as of the differentiating and articulating aspects with mental illness. The participants report that both mental illness and any type of disability are surrounded by prejudices and stereotypes that exclude and therefore discriminate. Conclusion. Professionals and students show a lack of information, training and awareness on the subject so that mental illness and psychosocial disability are two terms little related in the context of research. The medical model/social model articulation is urgent in the approach to the subject, giving relevance to the role that the context plays in the subject/society relationship from the rights approach.
\end{abstract}

\section{Key words}

Mental Health, Disability, Psychosocial, Social Exclusion, Mental Illness, Stigma (source: MeSH, NLM).

\section{Um olhar à deficiência psicossocial desde as ciências humanas, socinis e da saúde}

\section{Resumo}

A deficiência mental é um tema enquadrado pelo estigma que socialmente relega e estigmatiza ao sujeito, advertindo dum tipo de deficiência antes outrora com a intelectual: a deficiência psicossocial, hoje formalmente reconhecida pela Organização Mundial da Saúde. Objetivo. Compreender a concepção de transtorno mental e deficiência psicossocial, analisando o estigma e a exclusão como aspetos substantivos que configuram dito tipo de deficiência. Materiais e Métodos. Estudo qualitativo de tipo descritivo para o qual se aplicou em duas fases uma entrevista aberta estruturada e uma enquete a 51 pessoas: 20 profissionais das ciências humanas, sociais e da saúde e 31 estudantes de psicologia residentes na cidade de Ibagué. Resultados. Tanto quanto a percepção de estudantes como de profissionais, o transtorno mental e sua abordagem se remetem aos postulados do modelo médico tradicional. Existe desconhecimento da deficiência psicossocial, assim como dos aspetos diferenciadores e articuladores desta com o transtorno mental. Os participantes referem que tanto o transtorno mental como quaisquer tipo de deficiência estão rodeadas de prejuízos e estereótipos que excluem e por conseguinte discriminam. Conclusão. Profissionais e estudantes evidenciam ausência de informação, formação e sensibilização sobre o tema, pelo que o transtorno mental e deficiência psicossocial são dois termos pouco relacionados no contexto da pesquisa. Urge na abordagem da temática a articulação modelo médico/modelo social dando relevância ao papel que tem o contexto na relação sujeito/sociedade desde um enfoque de direitos.

\section{Palavias chave}

Saúde Mental, Deficiência, Psicossocial, Exclusão social, transtorno mental, Estigma (fonte: DeCS, BIREME). 


\section{Introducción}

La Organización Panamericana de la Salud (OPS) considera la discapacidad como el resultado de una relación entre la condición de salud de un individuo, sus factores personales y los elementos externos que determinan las circunstancias en las que se desenvuelve(1). Esta compleja relación entre condicionantes individuales y factores del entorno desencadenan en un conjunto de señalamientos y consecuencias en el individuo deficitario, dadas las dificultades que experimenta para acceder, hacer parte y participar plenamente en la vida social. Concepción que en la actualidad ha motivado el desarrollo de estudios sobre discapacidad más allá de los postulados médico-rehabilitadores clásicos, centrando su análisis en el modelo social y haciendo hincapié en la construcción social del sujeto y su relación bidireccional con el contexto, dotándolo de sentido e identidad en función de su devenir existencial inserto en un entorno sociocultural signado por los preceptos clasificatorios de la normalidad.

Y es que la discapacidad es un término que hace referencia tanto a la deficiencia o alteración en las funciones o estructuras corporales, las limitaciones o dificultades en la capacidad de realizar actividades, como a las restricciones vitales en la participación efectiva de las personas que poseen dicho déficit o alteración (2). De modo que esta noción genérica alude desde un marco taxonómico a las discapacidades cognitiva o intelectual, sensorial, física y psicosocial que, interpretadas como un constructo social, se anclan a otro concepto, el de normalidad que implícitamente define quien no lo es. Al respecto Ferrante y Ferreira refieren que la discapacidad tendría dos dimensiones: por un lado, una situación de discapacidad, donde esta establece atributos en un contexto; por otro lado, una condición de discapacidad, que pasa por la experiencia individual, donde los sujetos viven esos atributos, de forma tal que, en la experiencia de lo cotidiano, los esquemas y estructuras basales que significan la diada normal/patológico permiten interpretar la articulación entre situación y condición, pues a partir de tales esquemas se "inculca la concepción de cuerpo no legítimo" generando "unas prácticas y unos juicios sistemáticos" (3, p 85).

Entre tanto, el artículo que se presenta aborda específicamente la discapacidad psicosocial; un tipo de discapacidad que se inserta entre lo mental y lo social y que solo en la última década ha sido reconocida como tal, siendo justamente en la Convención sobre los Derechos de las Personas con Discapacidad en donde como instrumento jurídico se establece una distinción entre discapacidad intelectual y mental arguyendo características diferentes a las demás discapacidades. Empero, este tipo de discapacidad no es reconocida como tal en el contexto regional, por lo que la noción que se tiene en Colombia, en el Tolima particularmente, es ambiguo y obedece a cuestiones tales como falta de información, formación y sensibilización sobre el tema.

La discapacidad psicosocial es un proceso que aparece cuando el entorno no permite a una persona participar de la misma manera que todos a causa de un proceso o antecedente en salud mental (4), de modo que se advierte de una problemática principalmente social y poco reconocida catalogada como una enfermedad desamparada, y justamente por su desconocimiento denominada la cenicienta de las enfermedades y/o las discapacidades (5) dado que tal y como lo refiere la OMS, hace alusión a aquellos sujetos que con diagnóstico de trastorno mental han sufrido los efectos sociales negativos del estigma, la discriminación y la exclusión; por lo que el binomio tradicional normal/patológico, visto desde la discapacidad psicosocial, inculca una concepción de mente no legítima, generando indefectiblemente juicios y situaciones estigmatizantes como las antes mencionadas.

En tal sentido, los presupuestos básicos que condicionan el rotulo discapacidad psicosocial son, por un lado, la enfermedad mental y, por otro, 
la carga social que se impone al enfermo mental; puesto que para que se determine una situación de discapacidad psicosocial en un sujeto, este debe ser previamente diagnosticado por un profesional del área de la salud, quien al realizar una evaluación exhaustiva, rigurosa y pormenorizada de su estado de salud/enfermedad (acorde con los criterios establecidos en los manuales de clasificación de las enfermedades mentales) determina la configuración de trastornos como la depresión mayor, el trastorno bipolar, el trastorno obsesivocompulsivo, el trastorno límite de la personalidad, la ansiedad generalizada y la esquizofrenia, de forma tal que a partir del diagnóstico psicopatológico se pueda evidenciar la condición de sujeto psicosocialmente discapacitado a causa no exclusivamente del cuadro clínico que presenta, sino de su nivel de inserción y participación social y ciudadana; puesto que precisamente lo que le inculca la situación de discapacidad es el impacto que dicho diagnóstico genera en el ejercicio cotidiano del sujeto, inhabilitándolo para interactuar en la sociedad de forma adecuada (y aceptada) debido a las limitaciones que presenta en las actividades laborales, educativas y sociales a razón del estigma y la exclusión socioespacial que experimenta.

Por lo tanto, el punto de enunciación que se pretende expresar es la percepción distal que pueden evidenciar los profesionales (titulados y/o en formación) de diversas áreas del conocimiento en torno al tema. Por ejemplo, desde las ciencias de la salud se da cuenta de una postura cuya estructura basal es el modelo médico y el no reconocimiento del sujeto diagnosticado como una persona que puede padecer discapacidad psicosocial, situación que se presenta porque tradicionalmente la psicopatología ha sido abordada desde una perspectiva de intervención individual remitida al contexto clínico, mas no como un proceso de relación entre el individuo con la patología y las barreras actitudinales, comunicativas-semánticas, psicológicas, sociales y culturales, lo que acarrea situaciones tales como: actitudes negativas, falta de apoyo, discriminación, estigma y exclusión. Y desde las ciencias humanas y sociales lo psicosocial es un concepto susceptible de extrapolar a las diferentes situaciones y condiciones de discapacidad, pues se evidencia como una perspectiva de abordaje que no es exclusiva de aquellos con padecimientos de índole mental. No obstante, teórica y conceptualmente el termino da cuenta de la conjunción entre modelo médico y modelo social, pues surge de la incorporación del enfoque de derechos al enfoque médico para definir las restricciones ocasionadas por el entorno a aquellos cuya deficiencia es causada temporal o permanentemente por la mente.

Así que, de cara a la problemática expuesta, se reconoce formalmente la discapacidad psicosocial justamente para hacer mención a las personas que como resultado de su psicopatología han sido a lo largo de los años invisibilizadas y excluidas producto de las creencias, los imaginarios, la falta de apoyo, la falta de información y el aislamiento, a tal punto que desde la experiencia cotidiana de los sujetos como ciudadanos se evidencia un malestar constante en relación a los conflictos y tensiones vitales como la segregación espacial y la exclusión social de la población, a causa de las aparentes diferencias que aluden a percepciones, códigos y sensibilidades compartidas por un nosotros, que separan y marginan al otro, para de esta manera justificar su sometimiento y dependencia, negando, como indica Margulis (6) el derecho a ser diferente. Este planteamiento justifica indefectiblemente la incorporación del enfoque social y de derechos a la práctica médica y que sustenta lo expresado por el neurólogo Oliver Sacks (7) al hacer referencia a que más allá del qué de la enfermedad está el quién del enfermo, adoptando una postura crítica de los conceptos clásicos de salud y enfermedad.

En coherencia con lo expuesto, el proceso de investigación realizado a partir del cual surge el presente artículo, se soportó en el abordaje de cuatro categorías: la concepción de enfermedad mental, la concepción de discapacidad psicosocial 
y el estigma y la exclusión a causa de la enfermedad y/o la discapacidad, de las cuales, se encuentra escasa literatura en el contexto nacional y local; sin embargo, a partir de una revisión documental, se reconoce el desarrollo de algunos proceso de investigación e intervención que intentan una aproximación al tema y que fungen como antecedentes del estudio realizado y entre los cuales vale la pena resaltar los que a continuación se mencionan:

La evaluación de los conocimientos, actitudes, opiniones y creencias que tienen los adolescentes sobre la enfermedad mental, investigación desarrollada en el año 2015 que menciona cómo las creencias, la generalización errónea, los juicios de valores, las burlas o menosprecios y las actitudes negativas pueden conllevar a una asignación de etiquetas a los sujetos tales como "no se puede confiar en ellos, son vistos como criminales, delincuentes, violentos, asesinos o violadores" (8, p. 5), imaginarios que se derivan de la ignorancia, el prejuicio y la discriminación. Del mismo modo, la investigación titulada "Estudios sobre la enfermedad mental crónica: una revisión", realizada en el año 2016, indica que las personas que tienen un trastorno mental crónico manifiestan limitaciones en el desarrollo social, padeciendo momentos de sufrimiento tanto personal como familiar; describe también los retos a los que se enfrenta el cuidador principal de este tipo de pacientes, los cuales van desde la vigilancia de la adherencia al tratamiento hasta el afrontamiento de crisis propias de cada patología, así como la discriminación y el estigma social (9). Por otra parte, la implementación de la propuesta "Estrategias psicosociales utilizadas para el cambio de actitud hacia personas discriminadas por su discapacidad o trastorno mental", muestra que las estrategias más utilizadas y aplicadas en programas de orientación actitudinal son la educación y el contacto social, cuyos resultados positivos hacen referencia al logro en la modificación de las concepciones negativas y erróneas acerca de la diversidad y la diferencia (10).

Así, desde los antecedentes expuestos es posible afirmar la relación entre enfermedad mental y estigma social, pero sin incluir como tal el enfoque de discapacidad psicosocial en tanto proceso que construye e identifica al sujeto a causa justamente de dicho estigma, siendo este uno de los puntos de abordaje clave en la problemática: la relación triádica enfermedad mental - estigma social -discapacidad psicosocial. Al respecto, autores como Campo y Herazo (11) indican que el estigma es concebido como una secuencia lineal producto de las dinámicas socioculturales. Para Muñoz, Pérez, Crespo y Guillen (12), el estigma se pone de manifiesto en tres aspectos del comportamiento social: en primer lugar, existen los estereotipos considerados como ideas o modelos aprendidos por las personas en la sociedad, la cual generaliza a un grupo de población con características similares; cuando el hecho se aplica y se experimenta se pone en marcha el segundo aspecto, los prejuicios sociales, que a través de las emociones y las valoraciones danlugar al tercer aspecto denominado la discriminación efectiva, la cual incluye todos aquellos comportamientos de rechazo que colocan a la persona que padece una enfermedad mental en una condición evidentemente de detrimento a nivel social, llevándolo a configurar la discapacidad psicosocial.

Teniendo en cuenta lo anterior, el propósito del estudio que se presenta fue comprender, en primer lugar, la concepción de enfermedad mental, de discapacidad psicosocial y la relación entre los dos conceptos; y en segundo lugar, abordar el estigma y la exclusión como aspectos sustantivos que configuran la discapacidad, tanto desde la percepción de estudiantes de psicología, como de un grupo de profesionales de las ciencias humanas, sociales y de la salud en Ibagué, Tolima. 


\section{Materiales Y métodos}

Se realizó una investigación desde el enfoque cualitativo, de tipo descriptivo, la cual se desarrolló en dos fases, se tomó como población objeto de estudio, poruna parte, a profesionales de las ciencias humanas, sociales y de la salud, específicamente médicos generales, psiquiatras, psicólogos, trabajadores sociales y enfermeras, y por otra, estudiantes del programa de psicología de último periodo académico de la Universidad Nacional Abierta y a Distancia (UNAD) de la ciudad de Ibagué durante el año 2018, quienes participaron de manera voluntaria en el proceso investigativo, previa lectura y firma del consentimiento informado, de acuerdo con lo establecido por la República de Colombia y el Ministerio de Salud en la Resolución 008430 de 1993, por la cual se establecen las normas científicas, técnicas y administrativas para la investigación en salud. La investigación fue aprobada por un comité de investigadores y evaluada por un jurado.

Con el fin de obtener información fiable, el tipo de muestreo utilizado fue no probabilístico discrecional o también denominado muestreo intencional, el cual posibilitó la participación de la población por su perfil y el conocimiento sobre el tema de interés. Participantes: La muestra estuvo constituida por 51 personas (profesionales y estudiantes) considerados como agentes potenciales de conocimiento, puesto que en su labor desarrollan o desarrollarán procesos de evaluación, diagnóstico e intervención al grupo de población que constituye el tema de estudio, en ese sentido, los principales criterios de inclusión hicieron referencia a la pertenencia de los participantes a los grupos descritos (profesionales y/o estudiantes), ser residentes del municipio de Ibagué, expresar su voluntad de participar en el proceso investigativo y firmar el consentimiento informado.

Para cumplir con el objetivo de la investigación se tuvo en consideración la aplicación de técnicas e instrumentos empleados para conocer y explicitar el tema de interés, en consecuencia, se aplicó una entrevista semiestructurada constituida por 10 preguntas a partir de las cuales se indagaba sobre la percepción, conocimiento y significado que tienen y atribuyen los participantes a la discapacidad psicosocial y a la enfermedad mental en relación con la exclusión social y el estigma. La entrevista se realizó de forma flexible, aplicando las preguntas que fueron reformuladas en función de las respuestas de cada uno de los sujetos. A través de los significados y las definiciones personales se pretendió explicar y comprender el fenómeno de la discapacidad psicosocial. Se realizó un total de 51 entrevistas.

Adicionalmente, como segundo instrumento para la recolección de datos, se aplicó una encuesta con 5 opciones de respuesta tipo Likert, constituida por 17 ítems, de manera que se valoraron las actitudes y opiniones acerca de los estereotipos, prejuicio y discriminación, en torno a la enfermedad mental y la discapacidad psicosocial. Si bien, la investigación fue cualitativa, se utilizó este instrumento cuantitativo como apoyo al proceso realizado. La aplicación de las dos herramientas se llevó acabo en dos sesiones con el colectivo profesional y estudiantil, donde se compartió información sobre el objetivo de la investigación a realizar, además de nombrar de forma verbal el rigor ético con el que se manejaría la información aportada, resolviendo preguntas de los participantes.

Las dos herramientas mencionadas fueron desarrolladas con la disposición de cumplir con los objetivos de la investigación, así mismo, se seleccionaron por el fácil acceso y sistematización de los datos y por el análisis que se puede realizar de la información obtenida resaltando los hallazgos de forma objetiva y confiable. Los instrumentos fueron estructurados por el semillero de investigación Discapacidad Afecto y cognición vinculado al grupo Desarrollo Sociocultural, Afecto y Cognición de la Escuela de Ciencias Sociales, Artes y Humanidades ECSAH, de la Universidad Nacional Abierta y a Distancias (UNAD). 
El análisis de los datos se efectuó de forma inductiva e interpretativa, teniendo dos momentos: en el primero, se intercambiaron los hallazgos y se indagaron los temas objeto interés. En el segundo, los investigadores extrajeron la información de cada transcripción de la entrevista, plasmándola en una matriz donde se identificaron los temas principales, así mismo, se presentaron los resultados y datos de la encuesta, los cuales fueron analizados de acuerdo con las categorías de estudio previamente definidas, las cuales fueron: discapacidad psicosocial, enfermedad mental, exclusión social y estigma.

\section{Resultados}

\section{Características generales de los participantes}

La investigación conto con 51 participantes, de los cuales el $61 \%$ eran estudiantes de último periodo académico del programa de psicología con el 90\% de créditos académicos del programa aprobados, es decir 144 de un total de 160, y el $39 \%$ profesionales que se dedican a la prestación de servicios de diagnóstico, tratamiento, psicoeducación y rehabilitación en salud mental, ambulatoria y hospitalaria, específicamente: médicos, psicólogos, psiquiatras, enfermeras y trabajadoras sociales respectivamente, quienes laboran en diferentes instituciones prestadoras de salud tanto públicas como privadas de la ciudad de Ibagué.

Los principales hallazgos de la investigación fueron recolectados a través de la entrevista semiestructurada y la aplicación de la encuesta a cada participante. La información fue categorizada por los temas de interés: enfermedad mental, discapacidad psicosocial, estigma (estereotipo, prejuicio y discriminación) y exclusión social.

Tabla 1. Análisis de las categorías: enfermedad mental, discapacidad psicosocial, estigma y exclusión social de acuerdo con la percepción de profesionales y estudiantes en Ibagué, 2018

\begin{tabular}{|c|c|c|c|c|}
\hline \multirow[t]{2}{*}{ Categoría } & Tema 1 & Tema 2 & Tema 3 & Tema 4 \\
\hline & Enfermedad mental & $\begin{array}{c}\text { Discapacidad } \\
\text { psicosocial }\end{array}$ & Estigma & $\begin{array}{c}\text { Exclusión } \\
\text { social. }\end{array}$ \\
\hline Problemática & $\begin{array}{l}\text { Se tiene claridad frente } \\
\text { a la noción enfermedad } \\
\text { mental desde los } \\
\text { aportes del paradigma } \\
\text { médico tradicional } \\
\text { privilegiando la mirada } \\
\text { individualizada de } \\
\text { las problemáticas del } \\
\text { sujeto. }\end{array}$ & $\begin{array}{l}\text { La noción de } \\
\text { la discapacidad } \\
\text { psicosocial es } \\
\text { ambigua y no } \\
\text { se relaciona } \\
\text { directamente con la } \\
\text { enfermedad mental. } \\
\text { Los profesionales } \\
\text { evalúan el nivel de } \\
\text { ajuste psicosocial } \\
\text { del sujeto, sin tener } \\
\text { en cuenta el enfoque } \\
\text { de contextual y de } \\
\text { derechos que se da a } \\
\text { la discapacidad. }\end{array}$ & $\begin{array}{l}\text { Tanto estudiantes } \\
\text { como profesionales } \\
\text { reconocen el proceso } \\
\text { de estigmatización } \\
\text { que se da a los sujetos } \\
\text { con enfermedad } \\
\text { mental y a las } \\
\text { personas en situación } \\
\text { de discapacidad, } \\
\text { haciendo referencia } \\
\text { al prejuicio, la } \\
\text { discriminación, el } \\
\text { juicio de valor, las } \\
\text { reacciones negativas, } \\
\text { la asignación de } \\
\text { etiquetas y las } \\
\text { barreras actitudinales. }\end{array}$ & $\begin{array}{l}\text { Los } \\
\text { participantes } \\
\text { refieren la } \\
\text { exclusión social } \\
\text { como un factor } \\
\text { determinante } \\
\text { para la } \\
\text { vulneración de } \\
\text { los derechos } \\
\text { humanos. }\end{array}$ \\
\hline
\end{tabular}




\begin{tabular}{|c|c|c|c|c|}
\hline \multirow[t]{2}{*}{ Categoría } & Tema 1 & Tema 2 & Tema 3 & Tema 4 \\
\hline & Enfermedad mental & $\begin{array}{c}\text { Discapacidad } \\
\text { psicosocial }\end{array}$ & Estigma & $\begin{array}{l}\text { Exclusión } \\
\text { social. }\end{array}$ \\
\hline Definición & $\begin{array}{l}\text { Son patologías que } \\
\text { afectan la esfera } \\
\text { mental y generan } \\
\text { deterioro funcional, } \\
\text { las cuales deben ser } \\
\text { diagnosticadas por los } \\
\text { profesionales de la } \\
\text { salud. }\end{array}$ & $\begin{array}{l}\text { La discapacidad } \\
\text { psicosocial es una } \\
\text { condición que afecta } \\
\text { la capacidad del } \\
\text { sujeto para realizar } \\
\text { actividades de la } \\
\text { vida cotidiana, razón } \\
\text { por la cual cualquier } \\
\text { sujeto con algún } \\
\text { tipo de discapacidad } \\
\text { debe ser catalogada } \\
\text { como psicosocial. }\end{array}$ & $\begin{array}{l}\text { Los participantes } \\
\text { indican que el } \\
\text { estigma es un atributo } \\
\text { que devalúa al } \\
\text { individuo y genera } \\
\text { automáticamente } \\
\text { exclusión social } \\
\text { caracterizada por el } \\
\text { rechazo y aislamiento } \\
\text { del sujeto. }\end{array}$ & $\begin{array}{l}\text { Es un fenómeno } \\
\text { social que afecta } \\
\text { todas las áreas } \\
\text { de desarrollo del } \\
\text { sujeto y por lo } \\
\text { cual se vulneran } \\
\text { sus derechos. }\end{array}$ \\
\hline \multirow[t]{4}{*}{ Causas } & \multicolumn{4}{|c|}{$\begin{array}{l}\text { Los participantes desde su perspectiva indican que la falta de información, formación y } \\
\text { divulgación sobre el tema es un factor determinante para no tener claridad los aspectos } \\
\text { sustantivos que involucra la discapacidad psicosocial. }\end{array}$} \\
\hline & \multirow{3}{*}{\multicolumn{4}{|c|}{$\begin{array}{l}\text { En general algunos de los participantes están de acuerdo que las causas se expresan en } \\
\text { aspectos como: } \\
\text { Enfermedad mental y discapacidad psicosocial: primero en lo Biológico: Existe una } \\
\text { predisposición genética o de tipo orgánico que se refleja en cualquier etapa del desarrollo. } \\
\text { Segundo en lo Psicológico: Experiencias traumáticas o específicas que generan tensión, } \\
\text { consumo de sustancias psicoactivas, falta de adaptación en el medio y aceptación de la } \\
\text { discapacidad. } \\
\text { Estigma y exclusión: Falta de conocimiento por parte de las familias, no hay difusión } \\
\text { y sensibilización social y si proliferan creencias erróneas que se expresa a partir de } \\
\text { discursos compartidos en donde el poder mediático juega un papel importante en torno a } \\
\text { lo que es normal y anormal. }\end{array}$}} \\
\hline & & & & \\
\hline & & & & \\
\hline Consecuencias & \multicolumn{4}{|c|}{$\begin{array}{l}\text { Para los profesionales el desconocimiento sobre el tema produce una difusión equivocada } \\
\text { sobre el propio concepto, dando como resultado la "invisibilización" de los sujetos } \\
\text { y colectivos en situación de discapacidad psicosocial; en consecuencia, no se podrán } \\
\text { realizar procesos de intervención efectivos tendientes a mejorar la condición de } \\
\text { vida y a evitar problemáticas tales como las actitudes negativas, la falta de apoyo, la } \\
\text { discriminación, el prejuicio y el estereotipo que actualmente vive esta población en la } \\
\text { región. }\end{array}$} \\
\hline
\end{tabular}

Fuente: elaboración propia.

Por otra parte, desde una óptica comparativa fue posible evidenciar los puntos de acuerdo $\mathrm{y}$ diferencia entre profesionales en formación $\mathrm{y}$ titulados desde la postura epistemológica, en la cual se ubican sus disciplinas como ciencia social, humana y de la salud. 
Tabla 2. Comparativo según área de estudio disciplinar, 2018

\begin{tabular}{|c|c|c|}
\hline Abordaje comparativo & Ciencias Sociales y Humanas & Ciencias de la Salud \\
\hline Aspectos diferenciadores & $\begin{array}{l}\text { Los profesionales titulados y en } \\
\text { formación de las Ciencias Sociales y } \\
\text { Humanas (psicólogos, trabajadores } \\
\text { sociales y estudiantes de psicología) } \\
\text { refieren la importancia de abordar el } \\
\text { tema de la enfermedad mental tanto } \\
\text { desde el modelo médico con fines } \\
\text { clasificatorios, como desde el modelo } \\
\text { social en tanto problemática de abordaje } \\
\text { colectivo que requiere un proceso de } \\
\text { información, educación y comunicación } \\
\text { masivo a través del cual se busque } \\
\text { disminuir el estigma que rodea el tema. } \\
\text { Así mismo reconocen la importancia del } \\
\text { contexto y por tanto del modelo social } \\
\text { de la discapacidad para realizar procesos } \\
\text { de acompañamiento e intervención } \\
\text { colectivos haciendo alusión a que en } \\
\text { términos taxonómicos todos los tipos de } \\
\text { discapacidad son psicosociales. }\end{array}$ & $\begin{array}{l}\text { Los profesionales de las Ciencias } \\
\text { de la Salud (médicos generales, } \\
\text { psiquiatras y enfermeras) centran su } \\
\text { análisis en el modelo médico con } \\
\text { fines de rehabilitación individual } \\
\text { haciendo alusión a que los procesos } \\
\text { deben ser individualizados. Aunque } \\
\text { se asocia el estigma social con } \\
\text { la enfermedad mental no se hace } \\
\text { alusión al modelo social de abordaje, } \\
\text { pues se da cuenta de un proceso } \\
\text { de intervención exclusivo desde el } \\
\text { ámbito clínico e intrahospitalario. } \\
\text { Frente a la discapacidad psicosocial } \\
\text { no se reconoce el concepto y por tanto } \\
\text { no se asocia a la enfermedad mental } \\
\text { de forma tal que no se reconoce la } \\
\text { relación directa entre: } \\
\text { Enfermedad mental } \\
\text { Estigma social y exclusión } \\
\text { Discapacidad psicosocial }\end{array}$ \\
\hline
\end{tabular}

Los participantes refieren la necesidad de generar procesos de psicoeducación a partir de la psicopatología cuya función

Aspectos comunes principal sea orientar a los pacientes y sus familias sobre los procesos de enfermedad mental y las estrategias de abordaje. Por otra parte, manifiestan el escaso conocimiento en torno al tema de la discapacidad psicosocial en el contexto local y la necesidad de incluir en los planes de estudio de las profesiones a fines su reconocimiento y abordaje. Finalmente, consideran que se hace necesario un proceso de sensibilización social a partir del desarrollo de actividades tendientes a concienciar a la población sobre las problemáticas que acarrea la enfermedad mental y las consecuencias de la estigmatización.

Fuente: elaboración propia. 


\section{Enfermedad Mental}

En la categoría de la enfermedad mental, la mayoría de los participantes tienen claridad sobre el concepto, sus causas, consecuencias, síntomas y tratamiento de acuerdo con su formación disciplinar y profesional, aluden que el término tiene un origen clínico o psiquiátrico. En el siguiente apartado se observa alguna de las definiciones desde las voces de los participantes:

[...] la enfermedad mental es todo tipo de alteración a nivel emocional, cognitivo y comportamental.

[...] La enfermedad mental es una alteración cognitiva que afecta las emociones, los pensamientos y la conducta social de un individuo.

Ahora bien, existe una diferencia sustancial entre los dos grupos poblacionales que participaron en el proceso investigativo, la cual radica en la experiencia, manejo y abordaje por parte de los profesionales. Mientras los estudiantes no conocen el amplio abanico psicopatológico que se puede configurar a partir de un proceso de evaluación y diagnostico mental, los profesionales diferencian los trastornos mentales, así como los criterios diagnósticos de los mismos.

En cuanto a la relación existente entre enfermedad mental y discapacidad psicosocial, el grupo de participantes refiere que existe una diferencia entre los dos términos $\mathrm{y}$, por tanto, manifiestan que la enfermedad mental se presenta por una alteración biológica, mientras que la discapacidad psicosocial es producto de factores externos. Por otro lado, se evidencia que los profesionales y estudiantes refieren un mayor conocimiento y abordaje de trastornos tales como esquizofrenia, trastorno afectivo bipolar, depresión, ansiedad, demencia, trastorno obsesivo compulsivo, trastorno límite de la personalidad, los cuales son los de mayor prevalencia en la ciudad de Ibagué. Es de recalcar que los dos grupos manifiestan la importancia de abordar los temas estudiados, puesto que afirman que la enfermedad mental es un tema tabú que debe ser explorado, conocido $\mathrm{y}$ abordado desde una visión que trascienda el paradigma médico tradicional, con el fin de generar una conciencia colectiva sobre la población que vive esta condición, de tal forma que se suscite una verdadera inclusión social.

\section{Discapacidad Psicosocial}

Para los participantes el concepto de discapacidad psicosocial no es claro, se confunde con otro tipo de discapacidad y/o afirman no conocerlo, producto de la escasa información disponible, la poca sensibilización y falta de capacitación sobre el tema, por lo tanto, no lo asocian como una derivación del estigma y la exclusión que acarrea la enfermedad mental.

En la entrevista se evidencia la poca claridad del tema y desde las voces de los participantes se manifiesta que las causas que originan la discapacidad psicosocial se ve asociado con factores tales como lo biológico, lo ambiental, falta de comunicación, el entorno social, falta de inteligencia emocional de los ciudadanos, causa física, exclusión, falta de conocimiento, competencias ciudadanas, el rechazo inadecuado, falta de educación, estigma, aislamiento, estereotipos causados por la sociedad, pautas de crianza, inseguridad y factores estresantes.

Respecto a la opinión que tienen los participantes sobre los obstáculos que enfrentan las personas con discapacidad psicosocial, indican que el entorno social es el mayor obstáculo para cualquier tipo de discapacidad, puesto que se evidencian practicas tales como la discriminación, los estereotipos, los prejuicios y las creencias erróneas producto del desconocimiento del tema por parte de la sociedad. Otro obstáculo que describen los participantes es la falta de políticas públicas, lo cual no permite ofrecer bienestar en todas las dimensiones a esta población. 
Así mismo los participantes refieren la influencia del poder mediático en la percepción de realidad que socialmente se configura en torno a la enfermedad mental y la discapacidad en general, dado que tanto implícita como explícitamente se transmite una imagen errónea y negativa del tema, por lo cual se perpetúan falsas creencias en donde las personas "trastornadas" son causantes de vergüenza, rechazo, burla y miedo.

\section{Estigma: estereotipo, prejuicio y discriminación}

Para la recolección de información en torno al estigma se utilizó la encuesta, a partir de la cual se evidenció que las personas con enfermedad mental y/o discapacidad psicosocial son discriminadas por la sociedad, producto de las creencias sociales con relación a las afectaciones mentales, lo que incide en los procesos de no aceptación de las personas y la vulneración de sus derechos. La no integración de la persona con enfermedad mental puede derivar en una discapacidad psicosocial, esto debido a situaciones sociales como prejuicios, discriminación y exclusión.

De acuerdo con las respuestas de los participantes, la asignación de etiquetas sociales más comunes son: "son locos", "son personas peligrosas", "desubicados" y "enfermos", así mismo, genera reacciones emocionales negativas tales como "miedo", "temor", "desconfianza"y "señalamientos derechazo", los cuales desencadenandistintas formas de discriminación, lo que limita la configuración de relaciones y vínculos con una persona con algún diagnóstico de enfermedad mental. Por otro lado, los participantes están de acuerdo en que los diferentes medios de comunicación (radio, televisión, redes sociales, etc.) se constituyen en factor de riesgo para estigmatizar a cualquier grupo minoritario y para ello es importante tener en cuenta que la educación, el contacto social y los medios de comunicación pueden ser estrategias de intervención que promuevan el cambio de actitud poblacional con relación a la enfermedad mental y/o discapacidad psicosocial.

\section{Exclusión social}

La ausencia de participación de ciertos grupos poblacionales en la esfera social da lugar a la exclusión social, siendo el entorno un factor que impide y dificulta la participación plena a personas con un diagnóstico de enfermedad mental, puesto que se evidencian circunstancias que vulneran su condición humana dado el estigma social imperante. Estos procesos excluyentes generan en el individuo emociones negativas (deterioran su condición, su autoestima, su percepción de autoeficacia y ocasionan la aparición de sentimientos desalentadores), dando como resultado la discapacidad psicosocial.

En otro orden de ideas, los participantes manifiestan que la exclusión social puede repercutir no solamente en la persona con un diagnóstico de enfermedad mental, sino también a su familia y personas más cercanas, produciendo riesgos psicológicos como son los comportamientos auto excluyentes, alteraciones cognitivas, conductas auto punitivas o de violencia.

\section{Discusión}

Los hallazgos de este estudio permiten acercarse de forma cualitativa a la percepción que tienen estudiantes y profesionales de las ciencias sociales, humanas y de la salud sobre la discapacidad psicosocial en el municipio de Ibagué, departamento del Tolima; es importante reconocer que la discapacidad psicosocial es una derivación de la enfermedad mental, la cual se encuentra relacionado con el proceso de estigma y la exclusión social. Si bien se tienen conocimientos adecuados sobre los aspectos clínicos y de rehabilitación desde el ámbito ambulatorio y hospitalario sobre la enfermedad mental, se resalta que no se asocia el término con la discapacidad psicosocial, se comprobó que la población mencionada no tiene un concepto claro respecto a este tipo de discapacidad. 
Es importante resaltar que la percepción se desarrolla en todos los individuos, por lo que cada uno elabora una interpretación única e individual, derivada por influencias como los conocimientos, las experiencias, las expectativas adquiridas y circunstancias sociales. "En el proceso de percepción se pone en juego referentes ideológicos y culturales que reproducen y explican la realidad y que son aplicados a las distintas experiencias cotidianas para ordenarlas y transformarlas" (13, 49).

Aunque el concepto de discapacidad psicosocial no es nuevo a nivel internacional, a nivel nacional y regional es un tema reciente, puesto que no es conocida con profundidad ni con propiedad y no se encuentra ajustada a la realidad por cuestiones tales como falta de formación académica, divulgación y por la carencia de información sobre el mismo. La discapacidad psicosocial, a pesar de que no es nueva, es una de las más olvidadas y desatendidas, y está considerada como "invisible" debido a que no se puede detectar a simple vista (14).

De acuerdo con el marco del proyecto de Euro-social la discapacidad psicosocial se entiende: "como un producto social que resulta de la interacción entre una persona con un 'proceso psicoafectivo' y las barreras actitudinales y del entorno que la sociedad genera; y que, teniendo como base el estigma, el miedo y la ignorancia, limitan su participación plena en igualdad de condiciones con las demás" (4, p. 25). En cierto modo al no tener claridad sobre el tema, a algunos de los participantes se les dificulta reconocer las causas y consecuencias. Es decir que, en coherencia con lo expresado por la Organización de las Naciones Unidas (ONU), la discapacidad psicosocial hace referencia a una condición de vida temporal o permanente que afecta directamente las funciones mentales de interrelación de la persona y limita su capacidad para ejercer una o más actividades esenciales de la vida (15). Es importante mencionar que el impacto de la discapacidad psicosocial en el ejercicio cotidiano de la persona que la padece es muy alto, puesto que la inhabilita para interactuar con la sociedad de forma adecuada y aceptada, expresando limitaciones que se presentan en cualquier actividad. Cabe señalar que la discapacidad, según la OMS, es "un término general que abarca las deficiencias, las limitaciones de las actividades y las restricciones de la participación. Las deficiencias son problemas que afectan a una estructura o función corporal; las limitaciones de la actividad son dificultades para ejecutar acciones o tareas, y las restricciones de la participación son problemas para participar en situaciones vitales" (16).

Respecto a la exclusión social, es un concepto que los participantes conocen y lo identifican en la realidad cotidiana, evidenciando las barreras que impone la sociedad frente a las personas que padecen algún tipo de discapacidad, "el estigma, el estereotipo, el prejuicio y la discriminación se encuentran concebidos como una secuencia lineal, producto de las dinámicas socioculturales" (11, p. 244).

Por otro lado, los participantes indican que el estigma asociado con la enfermedad mental mantiene una fuerte relación negativa con los procesos de rehabilitación o en el impacto de la vida cotidiana, la investigación llevada a cabo por López, Rodríguez y Aparicio en el año 2008 muestra que el proceso del estigma involucra directamente tres aspectos como lo son los estereotipos "conjunto de creencias, en gran parte erróneas, que la mayoría de la población mantiene en relación en un determinado grupo social. Los Prejuicios son las predisposiciones emocionales, habitualmente negativas, que la mayoría experimenta con respecto a los miembros del grupo cuyas características están sujetas a creencias estereotipadas y finalmente la discriminación relacionada con el comportamiento social que se deriva del estereotipo y el prejuicio" (17, p. 47). Todo este conjunto de situaciones en relación con la enfermedad mental limita las funciones hasta un grado de incapacidad e impotencia en 
las actividades que realiza el sujeto diariamente, abarcando las actividades más frecuentes que realiza un sujeto y que están relacionadas con lo familiar, lo cotidiano, con las necesidades humanas, con la independencia y con el uso del tiempo (18).

Por tanto, se apela a la urgente necesidad de desarrollar propuestas de psicoeducación, formación y sensibilización sobre el tema abordado, dirigidas no solamente a estudiantes y profesionales, sino a la comunidad en general, con la finalidad de trascender de la orilla epistemológica tradicional de abordaje del tema centrada en el modelo médico, a una perspectiva social de la enfermedad mental y la discapacidad, precisamente porque una persona con un diagnóstico psicopatológico puede sufrir una discapacidad psicosocial (permanente o transitoria) máxime que tal y como lo demuestran Portilla Montoya y Dussan, la valoración emocional ocupa el mayor porcentaje frente a los demás motivos para asistir a centros de atención psicológica, lo que lleva a suponer que problemas de la vida se convierten en problemas psiquiátricos y estos a su vez se pueden convertir al sujeto en una persona con discapacidad psicosocial (19). Al respecto, desde los preceptos de la educación para la salud, es necesario trascender el modelo tradicional e insertar prácticas propias del modelo crítico (social). Desde el enfoque tradicional la salud se relaciona con una concepción positivista de la ciencia y una concepción biomédica que responsabiliza al individuo de las conductas insanas; son los factores individuales-conductuales y biológicos- los determinantes centrales de la enfermedad y el modelo crítico se sustenta en una perspectiva crítica de la ciencia y de la salud que concibe a esta última como producto de las condiciones sociales, culturales y ecológicas (20).

\section{Conclusiones}

Comprender la enfermedad mental, la discapacidad psicosocial y el estigma, y la exclusión social que rodean el tema, posibilita que profesionales y estudiantes puedan implementar estrategias coherentes en los procesos de diagnóstico, tratamiento, psicoeducación y rehabilitación integral de los pacientes con trastorno mental. Se evidenciaron los vacíos teórico-prácticos frente al conocimiento y abordaje de la discapacidad psicosocial a nivel local, haciéndose necesario un proceso de reflexión profunda y pormenorizada desde una visión integral que contribuya a modificar la percepción que tiene la sociedad respecto a este tipo de discapacidad, permitiendo ofrecer verdaderas oportunidades de inclusión social a las personas que la padecen.

El papel de los profesionales y los estudiantes de las ciencias humanas, sociales y de la salud es visibilizar el presente fenómeno de estudio para brindar un mejor apoyo, no solamente a la persona que vive un proceso de discapacidad psicosocial, sino también a su familia; de igual manera es indispensable desarrollar estrategias para evitar aquellas barreras que dificultan la participación plena y afectiva en la sociedad (estigma, estereotipo, prejuicio y discriminación) e incentivar la integración de la discapacidad psicosocial en los programas y los planes de desarrollo municipales para su abordaje. Indefectiblemente la enfermedad mental es una condición signada por el estigma y la exclusión que se refuerza socialmente desde la interacción y la comunicación mediática, por lo que es indispensable generar una concientización sobre el manejo de la información por parte de los medios de comunicación (prensa, radio y televisión), con la finalidad de que no sean agentes estigmatizadores y sí un aporte para el cambio de actitud que tiene la población sobre la enfermedad mental. 


\section{Referencios bibliográficos}

1. Organización panamericana de la salud. Clasificación internacional del funcionamiento, de la discapacidad y de la salud [Internet]. España: IMSERSO; 2001 [consultado 2019, febr. 02]. Disponible en: http://apps.who.int/iris/bitstream/10665/43360/1/9241545445

2. Instituto Colombiano de Bienestar Familiar. Lineamiento técnico para el programa especializado de atención a niños, niñas, adolescentes y mayores de 18 años con discapacidad del ICBF [Internet]. Colombia: ICBF; 2019 [consultado 2019, febr. 04]. Disponible en: https://www.icbf.gov.co/system/ files/procesos/lm7.p_lineamiento_tecnico_para_la_atencion_de_ninos_ninas_y_adolescentes_ con_discapacidad_con_derechos_amenazados_yo_vulnerados_v2.pdf

3. Ferrante C, Ferreira MĀ. Cuerpo y habitus: el marco estructural de la experiencia de la discapacidad. Intersticios [Internet]. 2011 [consultado 2020, sept. 10]; 5: 85-101. Disponible en: http://www. intersticios.es/article/view/8888

4. Ministerio de Justicia República del Paraguay. Protocolo De Atención A La Justicia De Personas Con Discapacidad Sicosocial [Internet]. España: Cyan, Proyectos Editoriales, S.A; 2014 [consultado 2019, febr. 06]. Disponible en: http://sia.eurosocial-ii.eu/files/docs/1416568572-PARAGUAY_10_ completo.pdf

5. Séptimo día. La enfermedad desamparada [Material audiovisual]. Colombia: Canal Caracol; 2013

6. Castellanos-García GE, Arenas A, Rojas-Delgado JE. Voces del silencio: la inclusión social desde una ruptura en la homogenización comunicativa oyente-sordo. Sciences Pi Journal [Internet]. 2018 [consultado 2019, febr. 10]; 4 (2): 26. Disponible en: https://www.sciencespijournal.com/ ediciones/2018-volumen-ii/art\%C3\%ADculo-3/

7. Rodríguez-Díaz S. Más allá de la discapacidad: reflexiones en torno a la relatividad de la organización sensorial. REDIS [Internet]. 2013 [consultado 2020, Sept. 11]; (2):51-58. Disponible en: https:// www.cedd.net/redis/index.php/redis/article/view/64/56

8. Ruiz-Sobrino E. Evaluación de los conocimientos, actitudes, opiniones y creencias que tienen los adolescentes sobre la enfermedad mental [Proyecto de investigación; PDF] [España]: Hospital general universitario Gregorio Marañón. 52P.

9. Rosas-Santiago F. Estudios sobre la Enfermedad Mental Crónica: una Revisión. Salud y Administración [Internet]. 2016 [consultado 2019, febr. 20]; 4 (9):25-30. Disponible en: http:// www.unsis.edu.mx/revista/doc/vol3num9/A3_Enfermedad_Mental.pdf

10. Delgado-Meza JA. Estrategias psicosociales utilizadas para el cambio de actitud hacia personas discriminadas por su discapacidad o trastorno mental. REDIS [Internet]. 2015 [consultado 2019, mzo. 5]; 3 (2):27-39. Disponible en: https://www.cedd.net/redis/index.php/redis/article/view/178/ pdf_39

11. Campo-Arias A, Herazo E. El complejo estigma-discriminación asociado a trastorno mental como factor de riesgo de suicidio. RCP [Internet]. 2015 [consultado 2019, Mzo. 10]; 44 (4):243-250. Disponible en: http://www.scielo.org.co/pdf/rcp/v44n4/v44n4a08.pdf

12. Muñoz M, Pérez-Santos E, Crespo M, Guillen AI. Estigma y enfermedad mental análisis del rechazo social que sufren las personas con enfermedad mental [Internet]. España: Editorial Complutense S A; 2009 [consultado 2019, Mzo. 20]. 1-388. Disponible en: https://webs.ucm.es/BUCM/ ecsa//9788474919806.pdf

13. Vargas-Melgarejo. Sobre el concepto de percepción. Alteridades [Internet]. 1994 [consultado 2019, mzo. 21]; 4 (8):47-53. Disponible en: https://www.redalyc.org/pdf/747/74711353004.pdf 
14. Comisión de Derechos Humanos de la Ciudad de México. Discapacidad psicosocial: invisibilidad inaceptable. Defensor revista de derechos humanos [Internet]. 2010 [consultado 2019, abr. 5]; (11):1-66. Disponible de: https://cdhcm.org.mx/wp-content/uploads/2014/05/dfensor_11_2010.pdf

15. Organización de las Naciones Unidas. Convención sobre los derechos de las personas con discapacidad [Internet]. 2007 [consultado 2019, my. 15]. Disponible en: https://www.un.org/esa/ socdev/enable/documents/tccconvs.pdf

16. Organización mundial de la salud [Sitio virtual] Discapacidades [Internet]. Ginebra: OMS; 2020; [Consultado 2019 jun. 11]. Disponible en: https://www.who.int/topics/disabilities/es/

17. López M, Laviana M, Fernández L, López A, Rodríguez AM. y Aparicio, A. La lucha contra el estigma y la discriminación en salud mental. Una estrategia compleja basada en la información disponible. Rev. Asoc. Esp. Neuropsiq [Internet]. 2008 [consulta 2019 jun. 20]; 28 (1):43-83. Disponible en: http://scielo.isciii.es/pdf/neuropsiq/v28n1/v28n1a04.pdf

18. Romero-Ayuso DM. Actividades de la vida diaria. anales de psicología [Internet]. 2007 [consultado 2019 jun. 21]; 23 (2): 264-271. Disponible en: https:/www.redalyc.org/pdf/167/16723213.pdf

19. Portilla-Maya S, Montoya-Londoño DM, Dussán-Lubert C, López-Jaramillo MR. Caracterización de los asistentes al centro de atención psicológica de la Universidad de Manizales. Hacia Promoc. Salud [Internet]. 2016 [consulta 2020 sept. 13]; 21(2): 127-141. Disponible en: https://www.redalyc. org/jatsRepo/3091/309149631013/309149631013.pdf

20. Peñaranda-Correa F, López-Ríos JM, Molina-Berrío, DP. La educación para la salud en la salud pública: un análisis pedagógico. Hacia. Promoc. Salud [Internet]. 2017 [consulta 2020 sept. 13]; 22 (1). DOI: $10.17151 / \mathrm{hpsal} .2017 .22 .1 .10$ 